\title{
Development and Validation of a High-Throughput Next-Generation Sequencing Workflow for SARS- CoV-2 Whole Genome Sequencing: Results from Over 65,000 Clinical Cases
}

\section{Sun Hee Rosenthal}

Quest Diagnostics (United States)

\section{Anna Gerasimova}

Quest Diagnostics (United States)

Rolando Ruiz-Vega

Quest Diagnostics (United States)

Kayla Livingston

Quest Diagnostics (United States)

Ron M. Kagan ( $\sim$ kaganr@questdiagnostics.com )

Quest Diagnostics (United States)

Yan Liu

Quest Diagnostics (United States)

\section{Ben Anderson}

Quest Diagnostics (United States)

\section{Renius Owen}

Quest Diagnostics (United States)

\section{Laurence Bernstein}

Quest Diagnostics (United States)

\section{Alla Smolgovsky}

Quest Diagnostics (United States)

\section{Dong Xu}

Quest Diagnostics (United States)

\section{Rebecca Chen}

Quest Diagnostics (United States)

\section{Andrew Grupe}

Quest Diagnostics (United States)

\section{Pranoot Tanpaiboon}

Quest Diagnostics (United States)

\section{Felicitas Lacbawan}

Quest Diagnostics (United States) 


\section{Research Article}

Keywords: SARS-CoV-2, COVID-19 control strategy, NGS, PCR method, standard ARTIC protocol

Posted Date: November 12th, 2021

DOI: https://doi.org/10.21203/rs.3.rs-997210/v1

License: (c) (1) This work is licensed under a Creative Commons Attribution 4.0 International License. Read Full License

Version of Record: A version of this preprint was published at Scientific Reports on February 8th, 2022. See the published version at https://doi.org/10.1038/s41598-022-06091-0. 


\section{Abstract}

Monitoring new mutations in SARS-CoV-2 provides crucial information for identifying diagnostic and therapeutic targets and important insights to achieve a more effective COVID-19 control strategy. Next generation sequencing (NGS) technologies have been widely used for whole genome sequencing of SARS-CoV-2. While various NGS methods have been reported, one chief limitation has been the complexity of the workflow, limiting the scalability. Here, we overcome this limitation by designing a workflow optimized for high-throughput studies. The workflow utilizes modified ARTIC network v3 primers for SARS-CoV-2 whole genome amplification. NGS libraries were prepared by a 2-step PCR method, similar to a previously reported tailed PCR method, with further optimizations to improve amplicon balance, to minimize amplicon dropout for viral genomes harboring primer-binding site mutation(s), and to integrate robotic liquid handlers. Validation studies demonstrated that the optimized workflow can process up to 2,688 samples in a single sequencing run without compromising sensitivity and accuracy and with fewer amplicon dropout events compared to the standard ARTIC protocol. We additionally report results for over 65,000 SARS-CoV-2 whole genome sequences from clinical specimens collected in the United States between January and September of 2021, as part of an ongoing national genomics surveillance effort.

\section{Introduction}

Coronavirus disease 2019 (COVID-19), caused by the severe acute respiratory syndrome coronavirus 2 (SARS-CoV-2), emerged in the Chinese province of Wuhan in November $2019^{1}$ and was declared a global pandemic by the World Health Organization after its rapid spread around the world (https://www.who.int/emergencies/diseases/novel-coronavirus-2019). Genomic surveillance has been employed to track the evolution of SARS-CoV-2 over the course of the pandemic, including the emergence of new variants that may affect viral transmissibility, infectivity, immune evasion, and vaccine efficacy ${ }^{2-}$ ${ }^{4}$. It further informs public health decisions by facilitating the tracking of SARS-CoV-2 transmission, outbreak detection, and contact tracking, and has been used to help trace the origin of the pandemic ${ }^{5-7}$. As of Sept 2021, more than three million SARS-CoV-2 genomic sequences worldwide have been deposited and made publicly available in the global initiative on sharing all influenza data (GISAID) database (https://www.gisaid.org/).

The initial SARS-CoV-2 genomic sequence was obtained through a metagenomic approach and confirmed by Sanger sequencing and $\mathrm{PCR}^{8-10}$. As the pandemic progressed, multiple NGS approaches have been utilized for SARS-CoV-2 sequencing, including shotgun metagenomics, hybrid capture enrichment, and amplicon-based sequencing ${ }^{11-14}$. Shotgun sequencing requires no prior knowledge of the targeted viral genome ${ }^{15}$ but is limited by requirements for a high viral load and a higher sequencing depth. Hybridization approaches target genomic regions of interest by using biotinylated probes and ensure a more complete profiling of regions of interest ${ }^{16}$. Since the initial release of the ARTIC SARS-CoV2 sequencing protocol early in the outbreak (Jan 22, 2020, https://artic.network/ncov-2019), amplicon- 
based sequencing has become the primary choice for many labs around the world and a number of commercial kits are also available ${ }^{14}$. This approach utilizes first-strand cDNA synthesis followed by genome amplification using viral genome specific primers to produce amplicons that are tiled across the entire genome. Sequencing adaptors and barcode indices are added, using either ligation or tagmentation-based approaches. However, the complexity of those workflows limits their scalability. Recently, Gohl et al (2020) ${ }^{17}$ reported a cost-effective and highly scalable tailed amplicon method for SARS-CoV-2 sequencing, which bypasses costly and time-consuming library preparation steps.

In this study, we report an automated, high-throughput workflow for SARS-CoV-2 whole genome sequencing utilizing a 2-step PCR NGS library preparation method with modified ARTIC v3 primers. Our workflow is similar to the method described by Gohl et al (2020) ${ }^{17}$, where the Illumina sequencing primerbinding sites were added to ARTIC v3 gene-specific primers for use in the subsequent PCR step to add the sequencing adapters and barcode sequences. However, we made further optimizations to improve amplicon coverage balance for high-multiplexing, to minimize amplicon dropout by employing touchdown $\mathrm{PCR}^{18}$, and to integrate automated liquid handlers for high-throughput surveillance studies. Validation studies performed on clinical specimens achieved robust whole genome sequencing coverage in a highly multiplexed setting with much reduced percent amplicon dropout even for the recently reported variants of concern. Using this method, we successfully converted our lower-throughput SARS-CoV-2 whole genome sequencing methodology to an automated, high-throughput process. We additionally report results for over 65,000 SARS-CoV-2 whole genome sequences from clinical specimens collected in the United States from January to September 2021 as part of the ongoing US Center for Disease Control (CDC) National SARS-CoV-2 Strain Surveillance (NS3) system (https://www.cdc.gov/coronavirus/2019ncov/variants/cdc-role-surveillance.html).

\section{Results}

\section{High-throughput NGS workflow optimization}

Our laboratory has developed an automated, high-throughput SARS-CoV-2 NGS workflow (Figure 1). This workflow utilizes a 2-step PCR NGS library preparation method: (1) gene-specific PCR to amplify the SARS-CoV-2 whole genome, using primers published by the ARTIC network, with modifications to add Illumina sequencing primer binding sites; and (2) index PCR to add specimen-specific barcoded sequencing adapters by fusion PCR using the Illumina sequencing primer binding sites. We first optimized primer pools to give even coverage across the SARS-CoV-2 genome. Gene specific primers were pooled into 4 pools (pool 1A, 1B, 2A, 2B, Supplemental Table S1), and positive patient specimens with RTqPCR cycle threshold (Ct) value of $24(n=11)$ were tested. To evaluate the coverage uniformity, we computed the average coverage of each amplicon at a normalized depth of 200,000 mapped reads. All 98 amplicons produced adequate coverage with a mean coverage of 973X (SD, 719; CV, 73.9\%) (Figure $2 A)$. However, some amplicons ( $n=7,3$ in the spike protein coding region) resulted in relatively lower coverage. When the same sample set was tested with a standard ARTIC v3 method, even coverage across 
the whole genome was achieved with a mean coverage of $1,390 X$ (SD, 658; CV, 47.3\%) at 200,000 mapped reads (Figure 2B).

Next, to improve coverage for these low-performing amplicons, we increased the corresponding primer concentration in the same primer pool or added the primer set to a different pool. When tested with RTqPCR positive patient specimens ( $C t 24, n=5$ ), the optimized primer pools improved the coverage of these low-performing amplicons by 2 - to 5 -fold, resulting in improved amplicon balance with a mean coverage of $893 X$ (SD, 514; CV, 57.6\%) (Figure 2C). For the same sample set, standard ARTIC v3 method achieved 1,470X mean coverage depth (SD, 692; CV 47.1\%) (Figure 2D).

As the SARS-CoV-2 genome has evolved, various mutations were found at the ARTIC v3 primer binding sites. Upon sequence analysis of 3,506 positive samples collected and processed in January and February of 2021 , we found that $96 \%(3,367 / 3,506)$ of the samples had at least one primer binding site mutation with a median of 3.0 (range: 1-9) mutations with the potential to impair PCR efficiency per affected sample (Supplemental Table S3).

To minimize adverse effects on sequencing coverage, we employed a touchdown PCR method by gradually reducing the annealing temperature from $65^{\circ} \mathrm{C}$ to $55^{\circ} \mathrm{C}\left(0.7^{\circ} \mathrm{C} /\right.$ second). We compared 79 samples with two different annealing temperature settings. With this approach, the percent amplicon dropout due to primer binding site mutations was decreased from $0.50-0.01 \%$ (Table 1 ). The percent amplicon dropout was calculated from the number of amplicons that did not generate coverage divided by the total amplicon number. Each dropout amplicon was manually reviewed for the presence of a mutation at the affected primer binding site. 
Table 1

Comparison of percent amplicon dropout by annealing temperature settings

\begin{tabular}{|c|c|c|c|}
\hline \multirow[t]{2}{*}{ Clade $^{1}$} & \multirow{2}{*}{$\begin{array}{l}\text { Samples } \\
\text { Tested (N) }\end{array}$} & \multirow{2}{*}{$\begin{array}{l}\% \text { Amplicon dropout: } \\
\text { Annealing at } 65^{\circ} \mathrm{C}\end{array}$} & \multirow{2}{*}{$\begin{array}{l}\text { \% Amplicon dropout: } \\
\text { Annealing at } 65^{\circ} \mathrm{C}-55^{\circ} \mathrm{C}^{2}\end{array}$} \\
\hline & & & \\
\hline $20 \mathrm{~A}$ & 12 & 0.34 & 0 \\
\hline $20 \mathrm{~B}$ & 10 & 0.51 & 0 \\
\hline $20 \mathrm{C}$ & 3 & 1.7 & 0.34 \\
\hline $20 \mathrm{G}$ & 24 & 0.21 & 0 \\
\hline 20H (Beta, V2) & 1 & 2.04 & 0 \\
\hline $20 \mathrm{I}$ (Alpha, V1) & 9 & 0 & 0 \\
\hline 21C (Epsilon) & 17 & 1.08 & 0 \\
\hline 21F (lota) & 3 & 0 & 0 \\
\hline Total & 79 & 0.5 & 0.01 \\
\hline
\end{tabular}

\section{Assay Precision}

We assessed intra-assay precision using 188 unique specimens with Ct values between 10 and 25 run in 3 replicates. Most (523/564; 92.8\%) sequences met quality control metrics (Figure 3A); 175/188 (93.1\%) unique samples met quality control requirements for 2 or more replicates and were utilized for lineage and clade comparison. All 175 samples had concordant clade and lineage assignments for all replicates (Table 2). Additionally, spike protein amino acid substitutions were analyzed for all 175 samples. On average, $7.3 \pm 2.9$ substitutions were detected per sample. There was $99.8 \%$ qualitative $(1,272 / 1,275,95 \%$ Cl: $99.3-99.9 \%$ ) agreement when the minority variant frequency was $>20 \%$ (Supplemental Figure S1A). The average \% CV for variant frequency between replicates was $0.5 \%$ (min CV: 0.0\%; max CV: 6.7\%). 
Table 2

Intra- and inter-assay concordance of clade and lineage assignment using the automated, high-throughput SARS-CoV-2 NGS workflow

\begin{tabular}{|c|c|c|c|}
\hline & Number of samples & & \\
\hline Clade/lineage $^{1}$ & Intra-assay precision & Inter-assay precision & Total \\
\hline 20A/B. 1 & 5 & 1 & 6 \\
\hline 20A/B.1.189 & 0 & 1 & 1 \\
\hline 20A/B.1.232 & 0 & 1 & 1 \\
\hline 20A/B.1.234 & 0 & 3 & 3 \\
\hline 20A/B.1.243 & 0 & 3 & 3 \\
\hline 20A/B.1.525 & 2 & 1 & 3 \\
\hline 20A/B.1.539 & 0 & 1 & 1 \\
\hline 20A/B.1.628 & 1 & 0 & 1 \\
\hline 20B/B.1.1 & 0 & 1 & 1 \\
\hline 20B/B.1.1.222 & 1 & 0 & 1 \\
\hline 20B/B.1.1.231 & 1 & 0 & 1 \\
\hline 20B/B.1.1.265 & 1 & 0 & 1 \\
\hline 20B/B.1.1.316 & 1 & 0 & 1 \\
\hline 20B/B.1.1.318 & 2 & 0 & 2 \\
\hline 20B/B.1.1.345 & 0 & 1 & 1 \\
\hline 20B/B.1.1.348 & 1 & 1 & 2 \\
\hline 20B/B.1.1.434 & 2 & 0 & 2 \\
\hline 20B/B.1.1.519 & 8 & 15 & 23 \\
\hline 20B/P.2 & 0 & 1 & 1 \\
\hline 20B/R.1 & 0 & 4 & 4 \\
\hline 20C/B.1 & 3 & 0 & 3 \\
\hline 20C/B.1.1 & 3 & 0 & 3 \\
\hline 20C/B.1.324 & 1 & 0 & 1 \\
\hline
\end{tabular}

${ }^{1}$ SARS-CoV-2 clades were assigned with Nextclade (https://clades.nextstrain.org/) and lineages were assigned with Pangolin (https://pangolin.cog-uk.io/). 


\begin{tabular}{|c|c|c|c|}
\hline & \multicolumn{3}{|c|}{ Number of samples } \\
\hline 20C/B.1.427 & 2 & 5 & 7 \\
\hline 20C/B.1.429 & 2 & 16 & 18 \\
\hline 20C/B.1.517 & 6 & 1 & 7 \\
\hline 20C/B.1.526 & 8 & 4 & 12 \\
\hline 20C/B.1.526.1 & 3 & 4 & 7 \\
\hline 20C/B.1.526.2 & 9 & 5 & 14 \\
\hline 20C/B.1.575 & 2 & 2 & 4 \\
\hline 20C/B.1.637 & 1 & 0 & 1 \\
\hline 20G/B.1.2 & 12 & 38 & 50 \\
\hline 20G/B.1.596 & 4 & 6 & 10 \\
\hline $\begin{array}{l}\text { 20l (Alpha, } \\
\text { V1)/B.1.1.7 }\end{array}$ & 77 & 44 & 121 \\
\hline 20I (Alpha, V1)/Q.4 & 2 & 0 & 2 \\
\hline 20I (Alpha, V1)/Q.8 & 1 & 0 & 1 \\
\hline $\begin{array}{l}\text { 20J (Gamma, } \\
\text { V3)/P.1 }\end{array}$ & 5 & 1 & 6 \\
\hline 21D (Eta)/B.1.525 & 2 & 0 & 2 \\
\hline 21F (lota)/B.1.526 & 5 & 0 & 5 \\
\hline 21H/B.1.621 & 2 & 0 & 2 \\
\hline Total & 175 & 160 & 335 \\
\hline
\end{tabular}

We then assessed inter-assay precision for 168 unique specimens with $\mathrm{Ct}$ values between 10 and 25 over 3 independent runs. Overall, 479/504 (95.0\%) sequences met quality control requirements (Figure 3B). Over $95 \%(160 / 168)$ of samples had valid sequence data for 2 or more replicates and were utilized for clade and lineage comparison. All 160 samples had concordant clade and lineage assignments (Table 2). On average, there were $5.9 \pm 3.2$ spike protein amino acid substitutions per sample with $99.5 \%(946 / 951$, 95\% Cl: 98.8-99.8\%) qualitative agreement between runs when the minority variant frequency was present in $>20 \%$ of the reads (Supplemental Figure S1B). The average $\%$ CV for the variant frequency between replicates was $0.9 \%$ (min CV: 0.0\%; max CV: 20.3\%). 


\section{Assay Sensitivity}

To demonstrate that our high-throughput workflow offers adequate sensitivity to yield complete SARSCoV-2 genomes, we determined the limit of detection. The limit of detection was defined as the highest $\mathrm{Ct}$ value that yielded valid sequence data with $\geq 97 \%$ SARS-CoV- 2 genome coverage for at least $95 \%$ of the specimens tested. A total of 39 unique samples with $\mathrm{Ct}$ values between 17 and 32 were serially diluted, yielding 186 samples with Ct values between 17 and 35 . The percent of samples that yielded $\geq 97 \%$ consensus sequence ranged between $91 \%$ and $100 \%$ up to a Ct value of 27 (Figure 4A). Only 50\% (10/20) and $25 \%(4 / 16)$ of samples with Ct values of 28 and 29 , respectively, passed. Of note, $100 \%(20 / 20)$ of samples with Ct values of 28 and $88 \%$ (14/16) of those with Ct values of 29 generated $>90 \%$ consensus sequence. Thus, $98.7 \%$ (147/149) of samples with Ct values less than 30 yielded $>90 \%$ consensus sequence (Figure 4B). When over $90 \%$ consensus sequence was generated, there was $100 \%$ concordance for clade and lineage assignments.

\section{Accuracy study}

Next, we assessed the accuracy of the consensus sequences generated by this workflow. Three commercial synthetic RNA positive controls (clade Alpha, Beta, and Gamma variants; Twist Bioscience) were tested 12 times each, using 4 replicates per set-up in 3 independent set-ups, by 3 different scientists. Of note, the synthetic RNA controls do not cover $100 \%$ of the SARS-CoV-2 genome, and amplicon dropout was expected. In 36 trials, the mean percent consensus sequence was $95.1 \%$ ( $\min 93 \%$; $\max 98 \%$ ). All controls resulted in $100 \%$ positive percent agreement for clade and lineage assignments ( $95 \% \mathrm{Cl}$ : $90.4 \%$, $100.0 \%$ ) and $100 \%$ for spike protein mutations that were detected when the minor allele frequency was over $20 \%$ (95\% Cl: $98.8 \%, 100.0 \%)$.

In addition, a total of 84 qRT-PCR negative samples were evaluated, in 3 independent set-ups using 28 negative samples per run, by 3 different scientists. All negative samples gave negative results, with mean amplicon coverage of 0.2 ( $\min 0.0 \%$, max $0.6 \%$ ) relative to the PCR plate average, yielding $100 \%$ samplelevel negative percent agreement (95\% Cl: 95.6\%, 100.0\%).

\section{Robustness study}

To assess assay robustness, a total of 2,688 samples (1,662 unique) were sequenced in a single Illumina NovaSeq sequencing run. Over $94.3 \%(2,416 / 2,562)$ of the total number of sequenced samples and $95.3 \%(1,584 / 1,662)$ of unique samples passed quality control (median coverage $2,478 \mathrm{X}$ ); all negative samples $(n=49)$ gave a negative result (median coverage $2.4 X,<0.1 \%$ relative coverage); and all positive controls $(n=29)$ gave the expected lineage and clade assignments. To monitor possible sample contamination, a total of 56 no-template controls (NTCs, 2 per 96 samples) were included. Although 54 of the 56 NTC gave a negligible number of reads ( $0.025 \%$ relative coverage to the plate mean), 2 NTCs showed $5.7 \%$ and $8.2 \%$ relative coverage indicating possible sample crossover. To assess the effects of 
the NTC contamination on assay accuracy, we compared the sequences to those obtained from the same samples $(n=1,446)$ previously sequenced using the standard ARTIC v3 workflow on Illumina MiSeq sequencers. Concordance was $99.9 \%(1,445 / 1,446)$ for clade, and $99.8 \%(1,444 / 1,446)$ for lineage assignment (Supplemental Table S4, S5). Moreover, there was $100 \%$ clade and lineage concordance for all positive samples processed in the same 384 -well plates with the 2 higher-coverage NTC wells. These results indicate that a low degree of NTC contamination is unlikely to interfere with accurate consensus sequence generation or with clade and lineage assignments.

Next, we analyzed the proportion of samples that generated $100 \%$ consensus coverage out of the samples that passed coverage QC (Table 3). With the optimized high-throughput workflow, over $95.9 \%$ of samples generated a complete consensus (range: $83.0-100 \%$ per clade). When the same set of samples were analyzed with a standard ARTIC v3 workflow, the proportion of samples with complete consensus sequence was much lower, $66.1 \%$ (range $0-100.0 \%$ per clade). In some variants (clades Beta, Delta, Epsilon, and Lambda) when sequenced with the ARTIC v3 workflow, amplicon dropouts were observed in almost all cases; dropout was resolved by employing our high-throughput workflow utilizing touchdown PCR. 
Table 3

Proportion of SARS-CoV-2 samples generating complete consensus sequence with the automated, highthroughput SARS-CoV-2 NGS workflow, by clade

\begin{tabular}{|c|c|c|}
\hline & \multicolumn{2}{|c|}{ Samples generating complete consensus sequence } \\
\hline & \multicolumn{2}{|c|}{ with each workflow, \% (Complete/Incomplete) ${ }^{1}$} \\
\hline Clade $^{2}$ & High-throughput workflow ${ }^{3}$ & ARTIC v3 workflow ${ }^{4}$ \\
\hline 19B & $100(3 / 3)$ & $0(0 / 3)$ \\
\hline $20 \mathrm{~A}$ & $83.0(44 / 53)$ & $62.2(33 / 53)$ \\
\hline $20 \mathrm{~B}$ & $96.2(102 / 106)$ & $59.4(63 / 106)$ \\
\hline $20 \mathrm{C}$ & $88.7(87 / 98)$ & $47.9(47 / 98)$ \\
\hline $20 \mathrm{G}$ & $96(168 / 175)$ & $81.1(142 / 175)$ \\
\hline $20 \mathrm{H}$ (Beta, V2) & $100(7 / 7)$ & $0(0 / 7)$ \\
\hline 201 (Alpha, V1) & $96.9(691 / 713)$ & $78.2(558 / 713)$ \\
\hline 20J (Gamma, V3) & $100(44 / 44)$ & $45.4(20 / 44)$ \\
\hline 21A (Delta) & $100(1 / 1)$ & $0(0 / 1)$ \\
\hline 21C (Epsilon) & $98.0(99 / 101)$ & $0.99(1 / 101)$ \\
\hline 21D (Eta) & $100(10 / 10)$ & $100(10 / 10)$ \\
\hline $21 \mathrm{~F}$ (lota) & $97.7(128 / 131)$ & $61.8(81 / 131)$ \\
\hline 21G (Lambda) & $100(1 / 1)$ & $0(0 / 1)$ \\
\hline $21 \mathrm{H}$ & $100(3 / 3)$ & $66.6(2 / 3)$ \\
\hline Total & $96.0(1,388 / 1,446)$ & $66.1(957 / 1,446)$ \\
\hline \multicolumn{3}{|c|}{${ }^{1}$ Complete consensus sequence as defined by obtaining $\geq 97 \%$ SARS-CoV-2 genome coverage. } \\
\hline \multicolumn{3}{|c|}{${ }^{2}$ SARS-CoV-2 clades were assigned with Nextclade (https://clades.nextstrain.org/). } \\
\hline \multicolumn{3}{|c|}{$\begin{array}{l}{ }^{3} \text { Samples were sequenced on the Illumina NovaSeq } 6000 \text { with the SP reagent kit using } 2 \times 251 \\
\text { cycles. }\end{array}$} \\
\hline${ }^{4}$ Samples were se & In the Illumina MiSeq with th & kit using 2 x 251 cycles \\
\hline
\end{tabular}

\section{Large-scale surveillance study}


From January to September 2021, over 65,000 SARS-CoV-2 qRT-PCR-positive or transcription-mediated amplification (TMA)-positive specimens were successfully sequenced in our laboratory. Between January and the end of May 2021, the sequencing libraries were constructed using an in-house validated modified ARTIC v3 protocol ${ }^{19}$; subsequently, libraries were constructed using the high-throughput workflow designed to improve scalability, reported in this study. Cumulatively, the Delta variant of concern (VOC) was detected at the highest frequency (35\%), because of the steep increase in prevalence of this variant after June 2021 (https://covid.cdc.gov/covid-data-tracker/\#variant-proportions). Viruses in clade Alpha were the second-most prevalent, accounting for $29 \%$ of specimens tested, reflecting their high prevalence before June 2021. Other variants being monitored (VBMs) were detected at much lower frequencies, including Epsilon (5\%), lota (5\%), and Gamma (4\%) (Table 4). Beta, Lambda, Eta, and Kappa variants were found in less than $1 \%$ of the specimens sequenced. 
Table 4

SARS-CoV-2 clade distribution of clinical cases analyzed between January and September 2021.

\begin{tabular}{|lll|}
\hline Clade $^{1}$ & Number cases & Percent \\
\hline $21 \mathrm{~A}$ (Delta) & 23,239 & 35.13 \\
\hline 20I (Alpha V1) & 19,022 & 28.76 \\
\hline $20 \mathrm{G}$ & 5,665 & 8.56 \\
\hline 21C (Epsilon) & 3,475 & 5.25 \\
\hline 21F (lota) & 3,259 & 4.93 \\
\hline 20C & 3,103 & 4.69 \\
\hline 20B & 2,558 & 3.87 \\
\hline 20J (Gamma V3) & 2,540 & 3.84 \\
\hline 20A & 1,929 & 2.92 \\
\hline 21H & 683 & 1.03 \\
\hline 20H (Beta V2) & 156 & 0.24 \\
\hline 19B & 141 & 0.21 \\
\hline 21G (Lambda) & 140 & 0.21 \\
\hline 21D (Eta) & 131 & 0.2 \\
\hline 20D & 68 & 0.1 \\
\hline 21B (Kappa) & 25 & 0.04 \\
\hline 20E (EU1) & 12 & 0.02 \\
\hline Total & 66,146 & 100 \\
\hline 1 SARS-CoV-2 clades were assigned with Nextclade (https://clades.nextstrain.org/). \\
\hline
\end{tabular}

We tracked the proportions of VBM and VOC variants on a weekly basis (Figure S2). The Epsilon variant was predominant (25.6\%) in January and February 2021, then subsequently declined in frequency as the Alpha and lota variants became more prevalent (65.3\% and $13.3 \%$, respectively) through May 2021 . The Delta variant subsequently emerged in May 2021, rapidly increasing in prevalence to reach $99.8 \%$ of all specimens sequenced by mid-September 2021. These trends were consistent with national variant trends as reported by the CDC (https://covid.cdc.gov/covid-data-tracker/\#variant-proportions).

\section{Discussion}


Pathogen whole genome sequencing has been employed to investigate cases of foodborne disease outbreaks, methicillin-resistant Staphylococcus aureus (MRSA) outbreaks, and outbreaks of other bacterial and fungal pathogens ${ }^{20}$. However, these studies were retrospective in nature. Rapid highthroughput viral genomic sequencing is being increasingly used for outbreak research and is changing the practice of epidemiology ${ }^{21}$. For example, advances in high-throughput sequencing facilitated nearreal time whole genome sequencing to investigate the 2014-2015 Ebola virus outbreak in West Africa ${ }^{22}$. In the present COVID-19 pandemic, rapid SARS-CoV-2 whole genome sequencing, combined with epidemiologic methods, identified or excluded nosocomial transmission events to directly affect outbreak management in real time ${ }^{23}$. Hospital-based whole genome sequencing set up in collaboration with public health departments has also facilitated real-time outbreak investigations and identification of transmission chains ${ }^{24}$. At a national level, the Coronavirus Disease 2019 (COVID-19) Genomics UK Consortium (COG-UK) has developed high-throughput sequencing and analysis workflows to generate hundreds of thousands of SARS-CoV-2 genomic sequences, enabling large-scale genomic epidemiology to inform public health response (https://www.cogconsortium.uk/). In the United States, the CDC established the SARS-CoV-2 Sequencing for Public Health Emergency Response, Epidemiology and Surveillance (SPHERES) initiative in the early stages of the pandemic to accelerate the use of real-time pathogen sequence data and molecular epidemiology for the COVID-19 pandemic response (https://www.cdc.gov/coronavirus/2019-ncov/variants/spheres.html).

Large scale and high-throughput whole genome sequencing of SARS-CoV-2 is essential to enable realtime epidemiologic surveillance and to better understand the evolution and spread of the pandemic, improve epidemiologic tracking, and enhance treatment and prevention strategies for COVID-19. To date, several groups have developed different protocols and workflows for SARS-CoV-2 whole genome sequencing by NGS. However, most of the published studies are small in study sample size, low in throughput or may require complex enzymatic steps ${ }^{12,25-30}$. In one study, the commercially available COVIDSeq test (Illumina) was scaled up to sequence 752 clinical samples in duplicate for a total of 1,536 samples and controls on a NovaSeq 6000 instrument using an S4 flow cell ${ }^{31}$. However, this study described only a single sequencing run and did not utilize an automated high throughput workflow. In the current study, we were able to use the lower output and less expensive SP flow cell to sequence over 2,600 samples.

Here, we report an automated, high-throughput workflow for SARS-CoV-2 whole genome sequencing optimized for large scale surveillance studies, utilizing a 2-step PCR NGS library preparation method with modified ARTIC protocol v3 primers. Validation studies performed on 1,711 unique clinical samples demonstrated high precision (100\% inter- and intra-assay precision) and accuracy (100\% PPA and 100\% NPA). Slightly reduced, but adequate sensitivity was achieved in comparison to the lower-throughput inhouse validated ARTIC v3 protocol: near complete consensus sequence was generated for samples of qRT-PCR Ct values less than 28 using the 2-step PCR workflow (Figure 4) and with samples of Ct less than 30 by the ARTIC v3 workflow ${ }^{19}$. 
For large-scale surveillance studies, integration of robotic liquid handlers is a crucial component. Various automation platforms are available for NGS library preparation. Upon evaluation of frequently utilized liquid handlers, the Agilent Bravo platform produced the most consistent results for our workflow in a 384-well format and was used for library preparation. As noted by Gohl et al (2020) ${ }^{17}$, a 2-step PCR method increased primer dimer formation which can result in lower sequencing quality. To remove any primer dimer present, we implemented 3 library cleaning steps: 1) Ampure bead clean up using a centrifugal washer in 384-well format; 2) manual Ampure bead clean up of a pooled library; 3 ) size selection using $1.5 \%$ gel on a BluePippin station prior to loading on the Illumina NovaSeq 6000 . With these stringent clean-up steps, our validation runs achieved over $90 \%$ (range $90.6 \%-93.4 \%$ ) high base call Phred quality scores (Q30 or higher).

The ARTIC amplicon-based whole-genome amplification of SARS-CoV-2 is considered a highly sensitive and accurate method and is currently employed by many laboratories around the world. One problem associated with amplification of the SARS-CoV-2 genome is amplicon dropout which can be caused when a given primer cannot stably hybridize to its specific complementary sequence binding site because of novel mutations at the primer binding site. As the SARS-CoV-2 genome has evolved, various mutations were found in ARTIC v3 primer binding sequence ${ }^{32}$. This type of dropout often requires redesign and revalidation of primers. To minimize amplicon dropout, we employed a touchdown PCR method which reduced amplicon dropout (Table 1). The effects of this improvement were most prominent for Beta, Delta, Epsilon, and Lambda clade specimens, achieving 100\% consensus sequence coverage in most trials (Table 3 ). On the other hand, for these 4 clades, when analyzed by the standard ARTIC v3 workflow, nearly all samples failed to achieve full consensus sequence coverage due to primer binding site mutations causing amplicon dropout (Table 3, Supplemental Table S6). We extended our analysis to samples collected between May and September of 2021 to show that the benefit of using the touchdown PCR method is not limited to the validation sample set. Similar result was observed for Beta, Delta, Epsilon, and Lambda clade specimens with $95 \%$ of samples generating a complete consensus sequence using the high-throughput workflow whereas only $2.9 \%$ of samples generated complete consensus sequence by the ARTIC v3 workflow (Supplemental Table S7). The ARTIC network has published a newer ARTIC v4 primer set, which was designed to avoid current high-frequency mutations, with the goals of minimizing amplicon dropout and producing high-accuracy variant calling. However, analytical validation is required prior to using this new primer set in surveillance studies. We envision that a touchdown PCR method can be easily adapted to any workflow and can avoid frequent primer redesign and validation.

The 2-step PCR method generated 30\% lower coverage compared to the ARTIC v3 method when the per sample coverage was averaged across all amplicons and normalized to 200,000 mapped reads per sample (Figure 2A, 2B). We postulate that this difference may be due to the higher primer dimer formation during the 2-step PCR as mentioned by Gohl et al (2020) ${ }^{17}$. Although the normalized coverage was reduced by approximately $30 \%$, on average, for the 2-step PCR method, the normalized coverage still exceeded the minimum coverage necessary to reliably generate a viral genome consensus sequence or to detect viral variants present in at least $50 \%$ of the reads (consensus calling threshold). Indeed, the 
absolute coverage obtained even when processing $>2,600$ samples on the NovaSeq sequencer (median coverage 2,478X) greatly exceeded the absolute coverage obtained for ARTIC v3 runs on the MiSeq sequencer (add median coverage 1,098X with batch size of 192). Therefore, these differences in normalized coverage have minimal practical impact on the ability of the 2-step PCR method to generate reliable whole genome consensus sequences or to detect variants.

In summary, we report on the development and the validation of an automated 2-step PCR, highthroughput NGS workflow for SARS-CoV-2 whole genome sequencing with high sensitivity and accuracy with much less amplicon dropout, which we have implemented for high-throughput SARS-CoV-2 genomic surveillance. The combination of automation and optimized bench and analysis workflows has enabled efficient, large-scale SARS-CoV-2 surveillance studies.

\section{Methods}

\section{Sample Collection}

We obtained remnant RNA stored at $-80^{\circ} \mathrm{C}$, extracted from deidentified clinical specimens previously tested for SARS-CoV-2 by qRT-PCR at Quest Diagnostics between February and August 2021. For workflow development, 16 positive samples with a Cycle threshold (Ct) value 24 were processed for sequencing. Analytical method validation studies were performed on a random set of 1,711 unique clinical specimens collected in March and April of 2021. In addition, 3 twist Synthetic SARS-CoV-2 RNA controls (Twist Bioscience, San Francisco, CA) and 24 negative samples ( $\mathrm{Ct}>50$ by qRT-PCR) were included for accuracy studies.

For surveillance studies, a random set of residual nasopharyngeal or oropharyngeal swab specimens from SARS-CoV-2 qRT-PCR positive or transcription mediated amplification (TMA) positive specimens, performed at Quest Diagnostics, were collected across the United States between January and September 2021. For SARS-CoV-2 qRT-PCR positive sample selection, $\mathrm{Ct}<25$ was applied for qualification. In accordance with ethical requirements and in accordance with US Department of Health and Human Services guidelines for the use of deidentified specimen remnants in research studies, specimens were deidentified prior to the study and were limited to remnant extracted RNA from discarded specimens previously submitted for commercial testing.

\section{Two-Step PCR library preparation}

For cDNA synthesis a mixture of $10.8 \mu$ l extracted RNA and $2.7 \mu$ l SuperScript IV VILO master mix (Thermo Fisher Scientific, Waltham, MA) was incubated at $25^{\circ} \mathrm{C}$ for $10 \mathrm{~min}$ followed by $50^{\circ} \mathrm{C}$ for $10 \mathrm{~min}$ and $80^{\circ} \mathrm{C}$ for $5 \mathrm{~min}$. To amplify the entire SARS-CoV-2 genome, four gene specific PCR reactions were performed using each of the four primer pools (Supplemental Table S1). The primers were based on the ARTIC v3 primer set ${ }^{33}$ with modifications to add Illumina sequencing primer binding sites: $5^{\prime}$ ACACTCTTTCCCTACACGACGCTCTTCCGATCT-3' was added to $5^{\prime}$ of all forward primers; 5'- 
GTGACTGGAGTTCAGACGTGTGCTCTTCCGATCT-3' was added to 5' of all reverse primers. Eleven of ARTIC v3 primers were replaced with primers reported by Itokawa et al $(2020)^{34}$ to avoid primer interactions: forward primers for amplicons 21, 36, and 89; reverse primers for amplicons 1, 9, 13, 15, 32, 38,76 , and 89 . For the gene specific PCR reactions, $1.5 \mu \mathrm{l}$ of cDNA was used in $15 \mu \mathrm{l}$ reaction mixture of Q5 Hot START DNA Polymerase kit (New England Biolabs, Ipswich, MA): $3 \mu \mathrm{l}$ of 5X buffer, $0.3 \mu \mathrm{l}$ of 10 mM dNTPs, $0.15 \mu$ l of polymerase, $2.5 \mu$ of $10 \mu \mathrm{M}$ primer pool (1A, 1B, 2A, or $2 \mathrm{~B})$, and $7.55 \mu \mathrm{l}$ nucleasefree water. The initial denaturation occurred at $98^{\circ} \mathrm{C}$ for $30 \mathrm{sec}$ followed by 30 cycles of $98^{\circ} \mathrm{C}$ for $15 \mathrm{sec}$, $65^{\circ} \mathrm{C}$ for $40 \mathrm{sec}, 55^{\circ} \mathrm{C}$ for $40 \mathrm{sec}$ (ramp rate of $0.7^{\circ} \mathrm{C} / \mathrm{sec}$ ), $72^{\circ} \mathrm{C}$ for $2.5 \mathrm{~min}$ and the final extension at $72^{\circ} \mathrm{C}$ for 2 min. The four PCR products for same clinical samples were combined and diluted 1:100 in nuclease-free water. The diluted product was amplified with index primers that add sample index as well as the Illumina sequencing adapters needed for sequencing on the Illumina sequencers. The forward and reverse index primers had the following structure:

forward, 5'-AATGATACGGCGACCACCGAGATCTACAC-[12nt-i5-index]-ACACTCTTTCCCTACACGACGCTC-3';

reverse, 5'-CAAGCAGAAGACGGCATACGAGAT-[12nt-i7-index]-GTGACTGGAGTTCAGACGTGTGC-3'.

For the index PCR, $3 \mu \mathrm{l}$ of the diluted gene specific PCR product was used in $15 \mu \mathrm{l}$ reaction mixture of KAPA HiFi HotStart ReadyMix (Kapa Biosystems, Wilmington, MA): $7.5 \mu \mathrm{l}$ of $2 \mathrm{X}$ buffer, $2 \mu \mathrm{l}$ of $5 \mu \mathrm{M}$ dualindexed primer mix, and $2.5 \mu \mathrm{l}$ nuclease-free water, using the following program: $98^{\circ} \mathrm{C}$ for $45 \mathrm{sec}$ followed by $15 \mathrm{cycles}$ of $98^{\circ} \mathrm{C}$ for $15 \mathrm{sec}$ and $60^{\circ} \mathrm{C}$ for $30 \mathrm{sec}, 72^{\circ} \mathrm{C}$ for $30 \mathrm{sec}$, and the final extension at $72^{\circ} \mathrm{C}$ for 1 $\min$.

The indexed products were purified with 0.7X Ampure XP beads (Beckman Coulter, Beverly, MA) on a BlueWasher 384-well format (BlueCatBio, Concord, MA) and quantified using Qubit dsDNA Broad-Range reagent (Thermo Fisher Scientific, Waltham, MA) on a Tecan Infinite F200 Pro, 384 well reader (Tecan, Männedorf, Switzerland). The purified products were pooled, 250ng per sample, into a single library using Hamilton STARlet (Hamilton, Reno, NV) and was concentrated with 0.6X Ampure XP beads and size selected using a $1.5 \%$ gel cassette on the BluePippin (Sage Science, Beverly, MA) according to the manufacturer protocol. The size selected library was used for loading on a MiSeq with 192 samples per run or on a NovaSeq 6000 with 1,536 or 2,688 samples per run.

\section{ARTIC v3 library preparation}

The CDNA synthesis and PCR steps were identical to the original ARTIC protocol with minor modifications. In brief, RNA samples were reverse transcribed using the SuperScript IV kit (ThermoFisher, Waltham, MA) and random hexamers. RNA samples with $\mathrm{Ct}<18$ were diluted 50 -fold (Ct 12-15) or 10 -fold (Ct 15-18) before use. In brief, $11 \mu \mathrm{l}$ RNA samples were mixed with $1 \mu \mathrm{l} 50 \mu \mathrm{M}$ random hexamers and $1 \mu \mathrm{l}$ $10 \mathrm{mM}$ dNTPs. The mixture was incubated for $5 \mathrm{~min}$ at $65^{\circ} \mathrm{C}$ and placed directly on ice. After 2-3 minutes, $7 \mu$ l enzyme mix containing $4 \mu \mathrm{l}$ XX Buffer, $1 \mu \mathrm{l} 0.1 \mathrm{M}$ DTT, $1 \mu \mathrm{l}$ RNaseOUT RNase inhibitor, and 1 $\mu$ l SuperScript IV reverse transcriptase was added to the samples. The reactions were incubated at $42^{\circ} \mathrm{C}$ 
for $50 \mathrm{~min}$, and at $70^{\circ} \mathrm{C}$ for $10 \mathrm{~min}$ followed by cooling to $4^{\circ} \mathrm{C}$. For the multiplex PCR reactions, $2.5 \mu \mathrm{l}$ of the cDNA was used in $25 \mu$ reaction mixture of Q5 Hot START DNA Polymerase kit (New England Biolabs, Ipswich, MA): $5 \mu \mathrm{l} 5 \mathrm{X}$ buffer, $0.5 \mu \mathrm{l} 10 \mathrm{mM}$ dNTPs, $0.25 \mu$ polymerase and $3.6 \mu \mathrm{l} 10 \mu \mathrm{M}$ primer pool 1 or pool 2, and $13.15 \mu$ nuclease-free water. The primer pools were based on the ARTIC v3 primer pool scheme with further optimization to enhance low-performing amplicons (Supplemental Table S2). The thermal program was identical to the original ARTIC protocol: $30 \mathrm{sec}$ polymerase activation at $98^{\circ} \mathrm{C}$ followed by 30 cycles of $15 \mathrm{sec}$ denaturing at $98^{\circ} \mathrm{C}$ and $5 \mathrm{~min}$ annealing and extension at $65^{\circ} \mathrm{C}$. The PCR products in pool 1 and 2 reactions for same clinical samples were combined and purified with $1 \mathrm{X}$ AMPure XP and quantified with Qubit Broad Range Kit on SpectraMax (Molecular Devices, San Jose, CA).

The purified PCR products were converted to Illumina sequencer-compatible libraries using the Twist Library Preparation kit (Twist Bioscience, San Francisco, CA). In brief, $300 \mathrm{ng}$ of purified PCR products in $15 \mu \mathrm{l}$ were end-repaired in $25 \mu \mathrm{l}$ of end repair reaction mixture: $2.5 \mu \mathrm{l} 10 \mathrm{X}$ buffer, $5 \mu$ l enzyme mix, and 2.5 $\mu \mathrm{l}$ water and incubated at $20^{\circ} \mathrm{C}$ for $30 \mathrm{~min}$, and at $65^{\circ} \mathrm{C}$ for $30 \mathrm{~min}$. Illumina adapter was ligated to the end-repaired mix by adding $25 \mu$ ligation master mix: $10 \mu \mathrm{l}$ XX buffer, $5 \mu$ l enzyme mix, $2.5 \mu \mathrm{l} 5 \mu \mathrm{M}$ adapter, and $7.5 \mu \mathrm{l}$ water and incubated at $20^{\circ} \mathrm{C}$ for $15 \mathrm{~min}$. The ligated products were purified using $0.8 \mathrm{X}$ Ampure XP beads for subsequent index PCR using KAPA HiFi HotStart ReadyMix in $50 \mu$ reaction volume with $6 \mu \mathrm{l} 5 \mu \mathrm{M}$ index primers using the following program: $98^{\circ} \mathrm{C}$ for $45 \mathrm{sec}$ followed by 8 cycles of $98^{\circ} \mathrm{C}$ for $15 \mathrm{sec}, 60^{\circ} \mathrm{C}$ for $30 \mathrm{sec}$, and $72^{\circ} \mathrm{C}$ for $30 \mathrm{sec}$, and the final extension at $72^{\circ} \mathrm{C}$ for $1 \mathrm{~min}$. The indexed products were purified using 1X Ampure XP beads, quantified using Qubit Broad range reagent, and normalized by pooling $400 \mathrm{ng}$ per sample. The pooled library was used for MiSeq loading with 192 samples per run.

\section{Sequencing}

For sequencing on a NovaSeq 6000 (Illumina, San Diego, CA), final library was diluted to $1700 \mathrm{pM}$ and PhiX was spiked at $18 \%$. The combined library was denatured with $0.2 \mathrm{~N} \mathrm{NaOH}$ and neutralized with $0.4 \mathrm{~N}$ Tris- $\mathrm{HCl}$ and sequenced on a NovaSeq 6000 SP Reagent kit using $2 \times 251$ cycles. For sequencing on a MiSeq (Illumina, San Diego, CA), the final library was diluted to $2 \mathrm{nM}$, and spiked in $20 \%$ PhiX, denatured with $0.2 \mathrm{~N} \mathrm{NaOH}$ and neutralized with $0.2 \mathrm{~N} \mathrm{HCl}$, and diluted with Illumina HT1 buffer to $10 \mathrm{pM}$ and sequenced using a MiSeq 600 cycle v3 kit, 2 x 251 cycles.

\section{Analysis}

For MiSeq runs, the paired-end fastq files generated by MiSeq Reporter 2.5.1.3 were used. For NovaSeq 6000 runs, bcl files generated by Illumina NovaSeq RTA v.3.4.4 were converted to fastq by using Illumina bcl2fastq software without lane splitting. For batch size of 1,536, the resulting fastq files were downsampled by $50 \%$. The fastq files were mapped to SARS-CoV-2 (MN908947.3 build) supplemented with human genome (GRCh37) reference sequence using BWA ${ }^{35}$. PCR primer sequences were trimmed from 
the mapped reads to MN908947.3 using iVar ${ }^{36}$. iVar was also used for variant calling and consensus sequence creation. Reads sorting and filtering were performed by SAMtools ${ }^{37}$. Coverage depth of each nucleotide position was determined with BEDTools ${ }^{38}$. Percent genome coverage was determined by counting the number of nucleotides meeting the minimum coverage requirement divided by the total SARS-CoV-2 genome length excluding the $5^{\prime}$ and $3^{\prime}$ ends not covered by the amplicon panel. For MiSeq results, a minimum of $10 x$ reads was required whereas for NovaSeq results, coverage equivalent to the number of mapped reads divided by 20,000 was required for consensus sequence generation and variant calling. For clade and lineage assignment, Nextclade version 1.3.0 and Pangolin version 3.1.11 with pangoLEARN 2021-08-24 were used.

\section{Analytical method validation}

Using the optimized workflow, we performed analytical method validation studies on 1,711 unique clinical specimens collected in March and April of 2021, along with positive and negative controls. A sequencing batch size of 1,536 or 2,688 was employed using 384-well plates (4 plates for the 1,536 batch size and 7 plates for the 2,688 batch size). When the batch size was 1,536 , the resulting fastq files were downsampled by $50 \%$ before analysis. For sample quality control, we set a minimum sequencing coverage threshold of $10 \mathrm{X}$ spanning at least $97 \%$ SARS-CoV-2 genome.

\section{Declarations}

\section{Contributions}

S.H.R, A.Ge, R.R.V and K.L. contributed equally to this study.

S.H.R. Designed the study, participated in viral genome sequencing, assay development and validation, data analysis and wrote the manuscript.

A.Ge. designed, developed and validated the bioinformatics analysis pipeline, performed genome sequence analysis for this study.

R.R.V. participated in study design and validation, viral genome sequencing, sample collection, assay validation and preparation and editing of the manuscript.

K.L. Designed, developed and validated the high-throughput laboratory automation used for viral genome sequencing in this study.

R.M.K designed and developed the viral genome database used in this study, implemented automated lineage and clade assignments to populate the database, performed data analysis and assisted with sample collection and edited the manuscript. 
B.A. assisted with high-throughput laboratory automation design and development and participated in viral genome sequencing.

R.O. provided scientific oversight, resources and guidance and contributed to the design of the study.

L.B., A.S., D.X., R.C. and A.Gr. Designed, developed and deployed the informatics infrastructure and software necessary to operationalize the assay pipeline and to provide and interface to the laboratory scientists who performed the testing and reporting.

P.T. and F.L. provided medical oversight and assisted in securing necessary resources and funding for this study.

All authors reviewed the manuscript.

\section{Additional Information}

\section{Data Availability}

NovaSeq runs and sequence IDs are listed in Supplementary Materials file "Sequenced_sample_list.xlsx". Fasta consensus sequences in compressed (zip) file format will be provided as supplementary materials upon manuscript acceptance.

\section{Competing Interests}

All authors are employees of Quest Diagnostics, a reference laboratory that performs commercial SARSCoV-2 diagnostic testing, including whole genome sequencing for surveillance projects.

\section{References}

1. Zhu, N. et al. A Novel Coronavirus from Patients with Pneumonia in China. New England Journal of Medicine 382, 727-733 (2020).

2. Boehm, E. et al. Novel SARS-CoV-2 variants: the pandemics within the pandemic. Clin Microbiol Infect 27, 1109-1117 (2021).

3. Harvey, W. T. et al. SARS-CoV-2 variants, spike mutations and immune escape. Nat Rev Microbio/ 19, 409-424 (2021).

4. Tao, K. et al. The biological and clinical significance of emerging SARS-CoV-2 variants. Nat Rev Genet. 17, 1-17 (2021).

5. Banerjee, A., Doxey, A. C., Mossman, K. \& Irving, A. T. Unraveling the Zoonotic Origin and Transmission of SARS-CoV-2. Trends Ecol Evol 36, 180-184 (2021). 
6. Ferdinand, A. S. et al. An implementation science approach to evaluating pathogen whole genome sequencing in public health. Genome Med 13, 121, doi:10.1186/s13073-021-00934-7 (2021).

7. Park, S. Y., Faraci, G., Ward, P. M., Emerson, J. F. \& Lee, H. Y. High-precision and cost-efficient sequencing for real-time COVID-19 surveillance. Sci Rep 11, 13669, doi:10.1038/s41598-021-93145-4 (2021).

8. Lu, R. et al. Genomic characterisation and epidemiology of 2019 novel coronavirus: implications for virus origins and receptor binding. The Lancet 395, 565-574, doi:https://doi.org/10.1016/S01406736(20)30251-8 (2020).

9. Wu, F. et al. A new coronavirus associated with human respiratory disease in China. Nature 579, 265269, doi:10.1038/s41586-020-2008-3 (2020).

10. Zhou, P. et al. A pneumonia outbreak associated with a new coronavirus of probable bat origin. Nature 579, 270-273, doi:10.1038/s41586-020-2012-7 (2020).

11. Nasir, J. A. et al. A Comparison of Whole Genome Sequencing of SARS-CoV-2 Using Amplicon-Based Sequencing, Random Hexamers, and Bait Capture. Viruses 12, doi:10.3390/v12080895 (2020).

12. Simonetti, M. et al. COVseq is a cost-effective workflow for mass-scale SARS-CoV-2 genomic surveillance. Nat Commun 12, 3903, doi:10.1038/s41467-021-24078-9 (2021).

13. Xiao, M. et al. Multiple approaches for massively parallel sequencing of SARS-CoV-2 genomes directly from clinical samples. Genome Medicine 12, 57, doi:10.1186/s13073-020-00751-4 (2020).

14. Chiara, M. et al. Next generation sequencing of SARS-CoV-2 genomes: challenges, applications and opportunities. Brief Bioinform 22, 616-630, doi:10.1093/bib/bbaa297 (2021).

15. Quince, C., Walker, A. W., Simpson, J. T., Loman, N. J. \& Segata, N. Shotgun metagenomics, from sampling to analysis. Nature Biotechnology 35, 833-844, doi:10.1038/nbt.3935 (2017).

16. Doddapaneni, H. et al. Oligonucleotide capture sequencing of the SARS-CoV-2 genome and subgenomic fragments from COVID-19 individuals. PLoS One 16, e0244468, doi:10.1371/journal.pone.0244468 (2021).

17. Gohl, D. M. et al. A rapid, cost-effective tailed amplicon method for sequencing SARS- CoV-2. BMC Genomics 4, 863 (2020).

18. Don, R. H., Cox, P. T., Wainwright, B. J., Baker, K. \& Mattick, J. S. 'Touchdown' PCR to circumvent spurious priming during gene amplification. Nucleic Acid Res 19, 4008 (1991).

19. Rosenthal S et al. in The Journal of Molecular Diagnostics Vol. 11 (2020).

20. Le, V. T. \& Diep, B. A. Selected insights from application of whole-genome sequencing for outbreak investigations. Curr Opin Crit Care 19, 432-439, doi:10.1097/MCC.0b013e3283636b8c (2013).

21. Oude Munnink, B. B. et al. The next phase of SARS-CoV-2 surveillance: real-time molecular epidemiology. Nat Med 27, 1518-1524, doi:10.1038/s41591-021-01472-w (2021).

22. Wohl, S., Schaffner, S. \& Sabeti, P. Genomic Analysis of Viral Outbreaks. Annual Reviews 3, 173-195 (2016). 
23. Francis, R. V. et al. The impact of real-time whole genome sequencing in controlling healthcareassociated SARS-CoV-2 outbreaks. J Infect Dis, doi:10.1093/infdis/jiab483 (2021).

24. Walker, A. et al. Characterization of SARS-CoV-2 infection clusters based on integrated genomic surveillance, outbreak analysis and contact tracing in an urban setting. Clin Infect Dis, doi:10.1093/cid/ciab588 (2021).

25. Charre, C. et al. Evaluation of NGS- based approaches for SARS-CoV-2 whole genome characterisation. Virus Evol 6, veaa075 (2020).

26. Li, J. et al. Rapid genomic characterization of SARS-CoV-2 viruses from clinical specimens using nanopore sequencing. Sci Rep 10, 17492 (2020).

27. Li, T. et al. Rapid High-Throughput Whole-Genome Sequencing of SARS-CoV-2 by Using One-Step Reverse Transcription-PCR Amplification with an Integrated Microfluidic System and Next-Generation Sequencing. Clin Microbiol Infect 59, e02784-02720 (2021).

28. Nasir, J. A. et al. A Comparison of Whole Genome Sequencing of SARS-CoV-2 Using Amplicon-Based Sequencing, Random Hexamers, and Bait Capture. Viruses 12, 895 (2020).

29. Plitnick, J. et al. Whole Genome Sequencing of SARS-CoV-2: Assessment of the lon Torrent AmpliSeq Panel and Comparison with the Illumina-MiSeq ARTIC Protocol. J Clin Microbiol, Jcm0064921, doi:10.1128/jcm.00649-21 (2021).

30. Stüder, F., Petit, J. L., Engelen, S. \& Mendoza-Parra, M. A. Real-time SARS-CoV-2 diagnostic and variants tracking over multiple candidates using nanopore DNA sequencing. Sci Rep 11, 15869 (2021).

31. Bhoyar, R. C. et al. High throughput detection and genetic epidemiology of SARS-CoV-2 using COVIDSeq next-generation sequencing. PLoS One 16, e0247115, doi:10.1371/journal.pone.0247115 (2021).

32. Kuchinski, K. et al. Mutations in emerging variant of concern lineages disrupt genomic sequencing of SARS-CoV-2 clinical specimens. medRxiv 06.01.21258181 (2021).

33. Tyson, J. R. et al. Improvements to the ARTIC multiplex PCR method for SARS-CoV-2 genome sequencing using nanopore. bioRxiv (2020).

34. Itokawa, K., Sekizuka, T., Hashino, M., Tanaka, R. \& Kuroda, M. Disentangling primer interactions improves SARS-CoV-2 genome sequencing by multiplex tiling PCR. Plos One 15, e0239403, doi:https://doi.org/10.1371/journal.pone.0239403 (2020).

35. Li, H. \& Durbin, R. Fast and accurate short read alignment with Burrows-Wheeler transform. Bioinformatics 25, 1754-1760 (2009).

36. Grubaugh, N. D. et al. An amplicon-based sequencing framework for accurately measuring intrahost virus diversity using PrimalSeq and iVar. Genome Biology 20 (2019).

37. Li, H. et al. The Sequence Alignment/Map formt and SAMtools. Bioinformatics 25, 2078-2079 (2009). 
38. Quinlan, A. \& Hall, I. BEDTools: a flexible suite of utilities for comparing genomic features. Bioinformatics 26, 841-842 (2010).

\section{Figures}

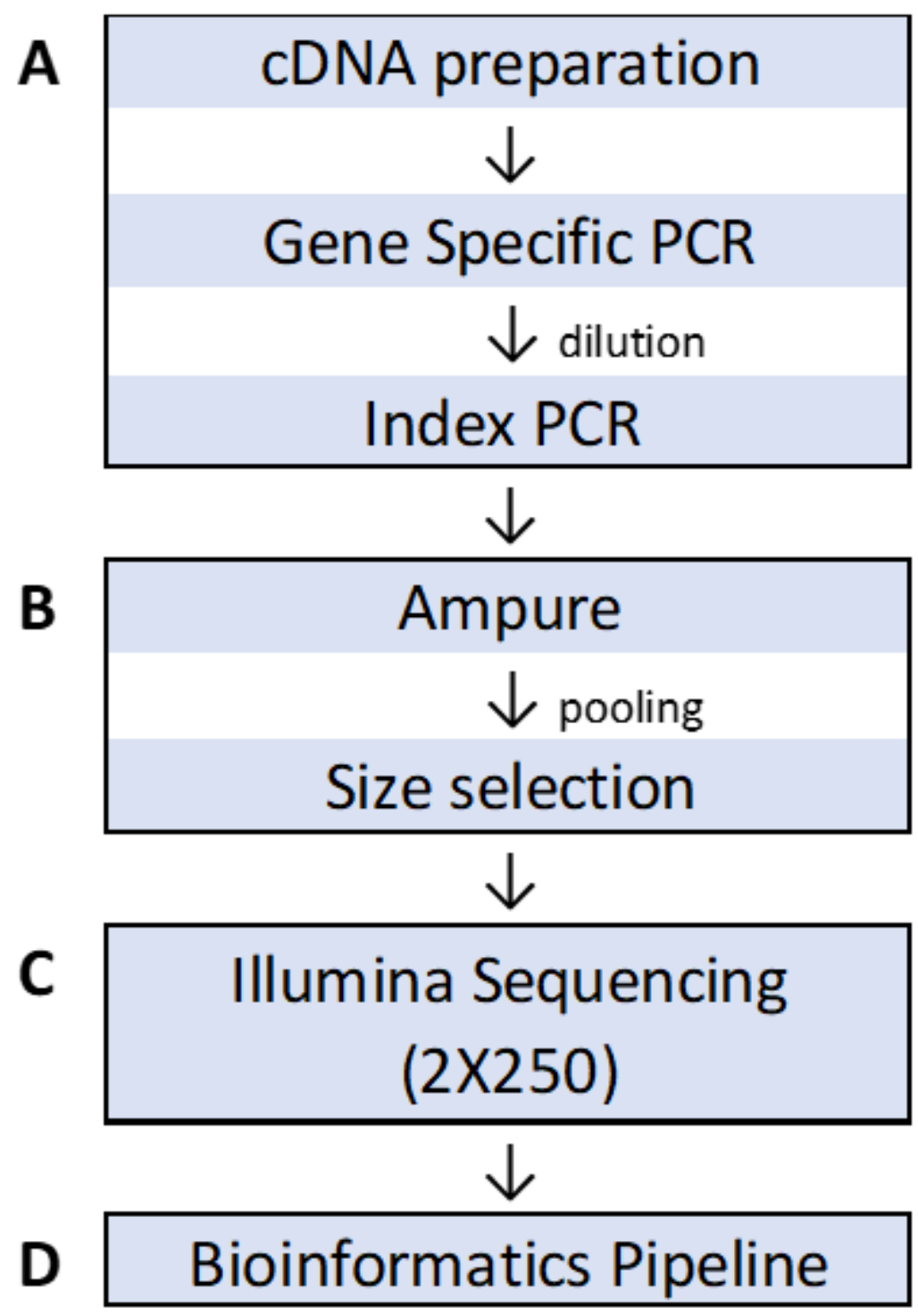

Figure 1

High-throughput NGS workflow. Sequencing library preparation was performed on robotic liquid handlers. A. Extracted RNA of SARS-CoV-2 positive specimens were converted to CDNA and subjected to touchdown PCR with primers published by the ARTIC network with modifications to add Illumina sequencing primer binding sites. Specimen-specific indexed sequencing adapters were added by subsequent fusion PCR using the primer binding sites. Agilent Bravo was used for each process. B. The final indexed PCR products were purified using Ampure XP beads using BlueCat BlueWasher, pooled into a single library using Hamilton Starlet, and size selected using Sage Science Blue Pippin. C. The final library was sequenced on an Illumina NovaSeq 6000. D. An in-house developed bioinformatics pipeline 
was utilized to generate consensus genomes and for variant calls relative to the reference genome MN908947.3, Wuhan-Hu-1.

A 10000

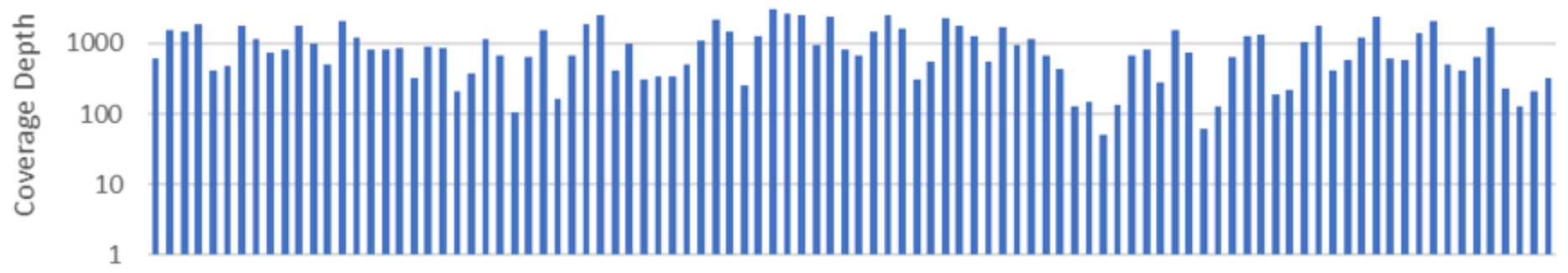

B

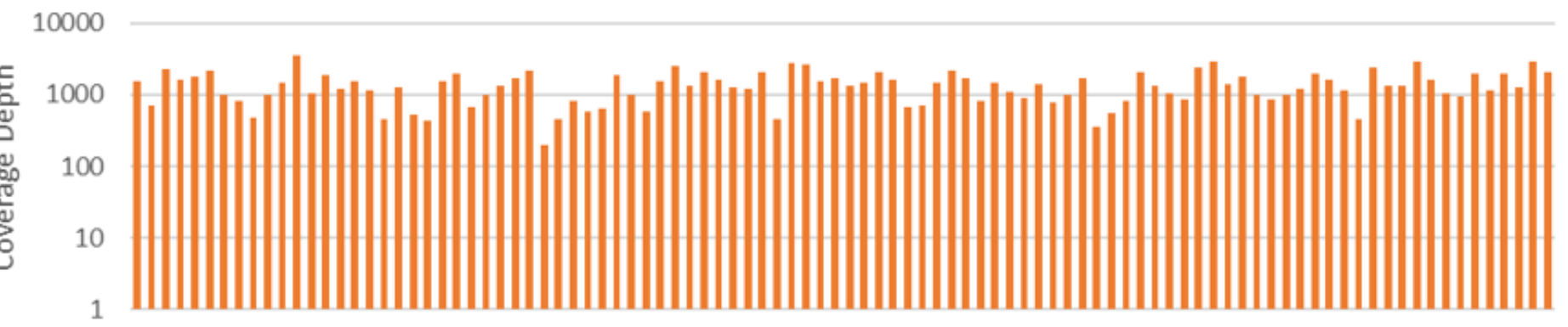

C 10000

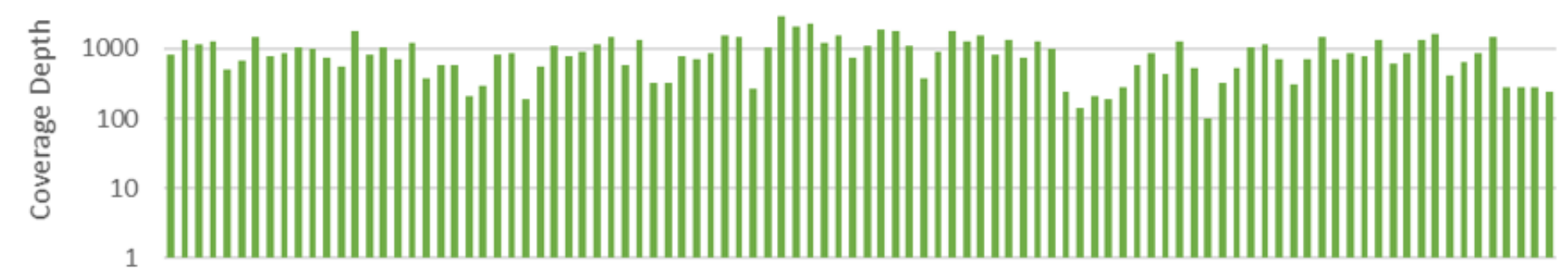

D 10000

1 c|l||

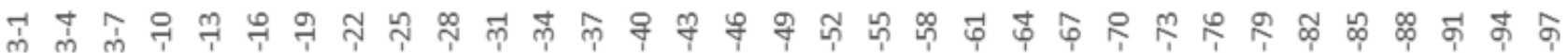

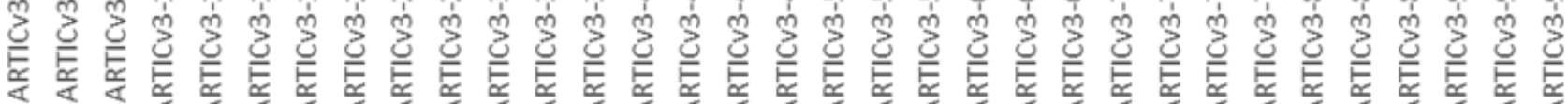
Amplicon

Figure 2

Comparison of amplicon coverage balance. A depth plot of (A) using equimolar primer pools, low performing amplicons were observed; (C) optimized modified primer pools, coverage of low performing amplicons was improved; (B, D) ARTIC v3 standard primers with even coverage. A same clinical sample set was used for plot $A$ and $B(C t 24, n=11)$; and $C$ and $D(C t 24, n=5)$. All samples were normalized to 200,000 mapped reads for comparison. 

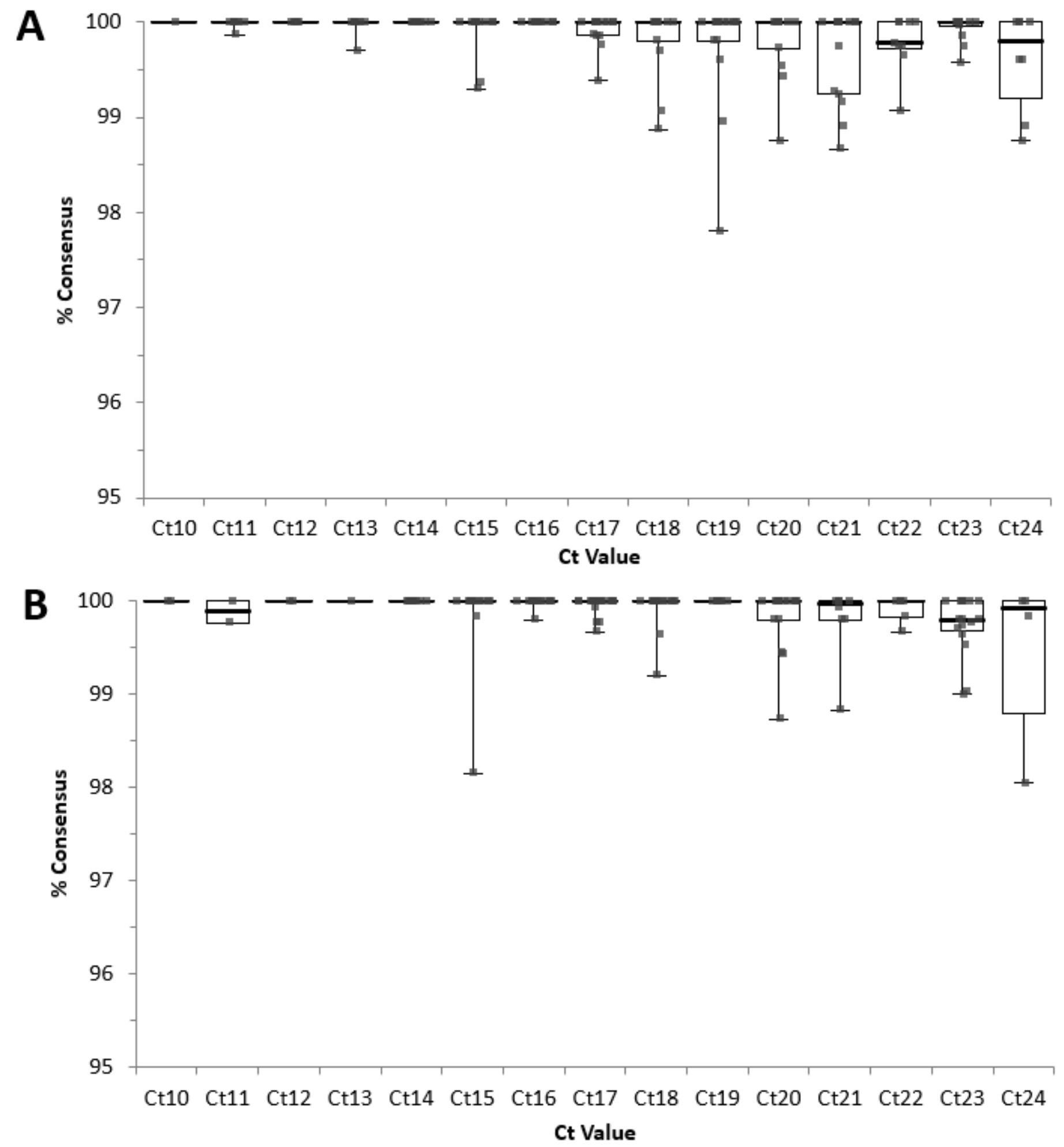

Figure 3

Percent genome coverage for intra-assay (A) and inter-assay (B) precision samples. Square dots indicate the average percent coverage of the SARS-CoV-2 genome for each replicate. The box plot shows the medians, interquartile ranges (IQR) and 1.5x IQR at each Ct value. Ct values were rounded down. 
A

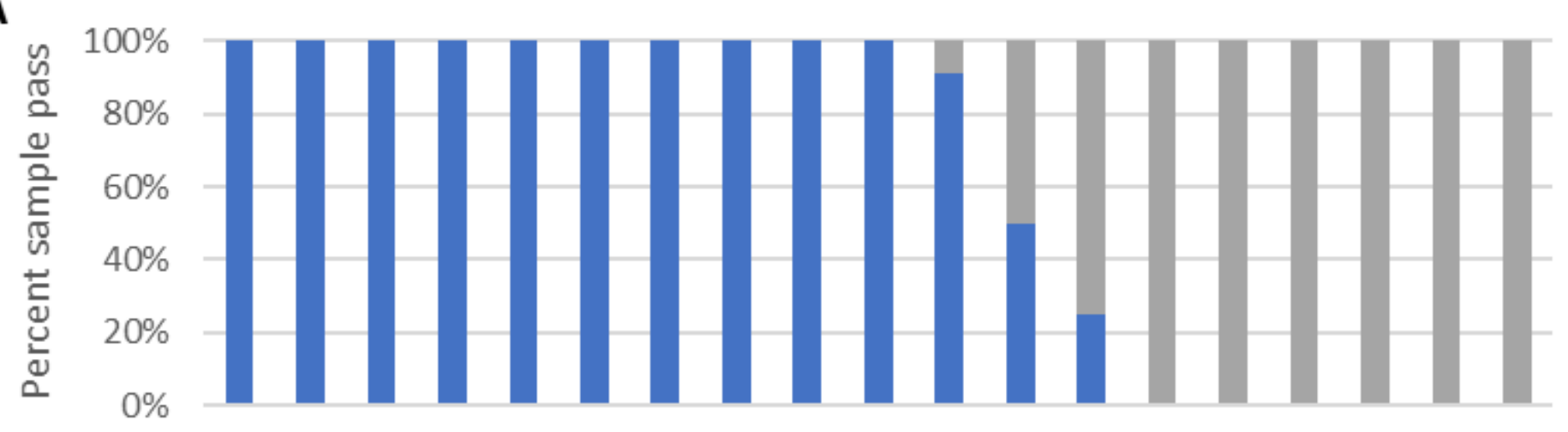

B

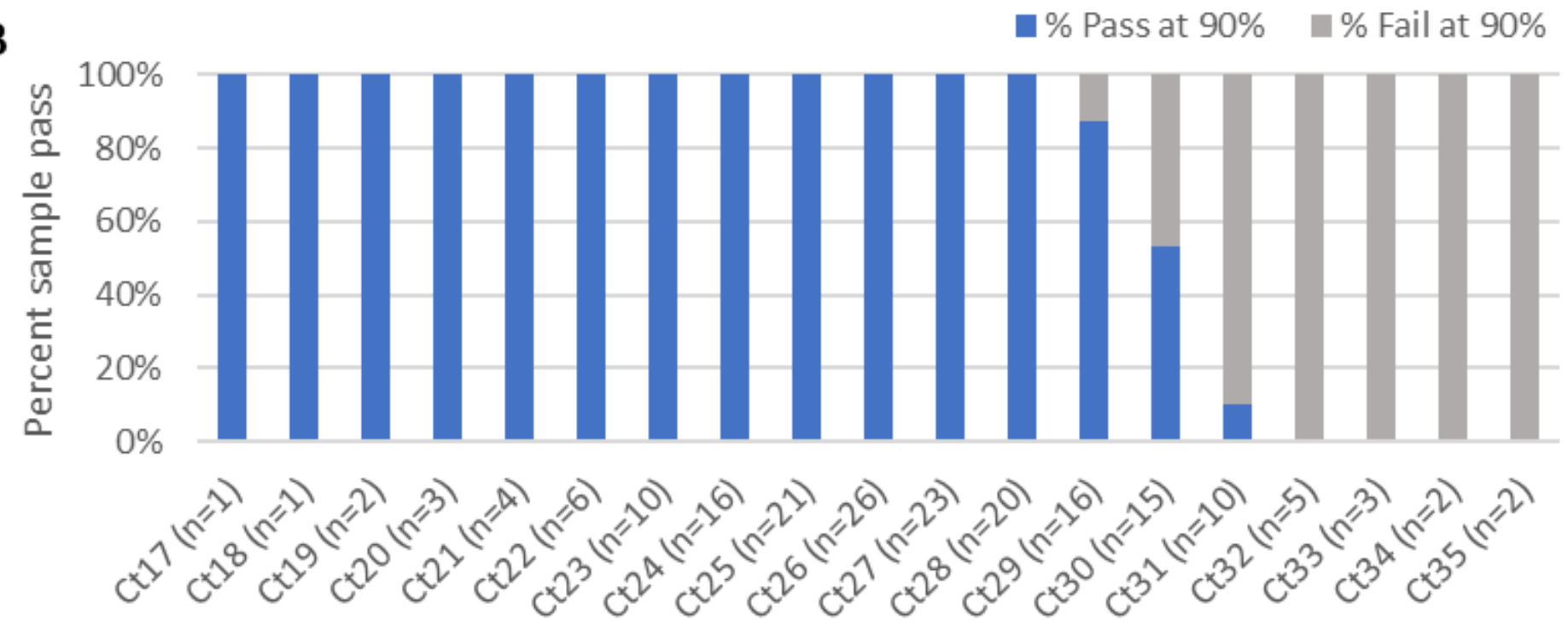

Figure 4

Sequencing pass rate (\%) for $\mathrm{Ct}$ values between 17 and 35 . Percent sample pass at over $97 \%$ consensus sequence coverage (A), and at over $90 \%$ consensus sequence coverage (B). Sample numbers tested per $\mathrm{Ct}$ are indicated in parentheses. $\mathrm{Ct}$ values were rounded down.

\section{Supplementary Files}

This is a list of supplementary files associated with this preprint. Click to download.

- Sequencedsamplelist.xlsx

- SupplementaryMaterials1.1.docx 